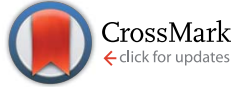

Cite this: J. Mater. Chem. C, 2014, 2 , 8990

Received 10th August 2014

Accepted 18th September 2014

DOI: $10.1039 / \mathrm{c} 4 \mathrm{tc} 01776 \mathrm{e}$

www.rsc.org/MaterialsC

\section{Polymerisable squaramide receptors for anion binding and sensing $\dagger$}

\author{
P. Manesiotis, * A. Riley and B. Bollen
}

A novel series of polymerisable squaramides has been synthesised in high yields using simple chemical reactions, and evaluated in the binding of anionic species. These vinyl monomers can be used as functional building blocks in co-polymerisations with a plethora of co-monomers or cross-linkers, grace to their compatibility with free-radical polymerisation reactions. Aromatic substituted squaramides were found to be the strongest receptors, while binding of certain anions was accompanied by a strong colour change, attributed to the de-protonation of the squaramide. The best performing squaramide monomers were incorporated in molecularly imprinted polymers (MIPs) targeting a model anion and their capacities and selectivity were evaluated by rebinding experiments. Polymers prepared using the new squaramide monomers were compared to urea based co-polymers, and were found to contain up to $80 \%$ of the theoretical maximum number of binding sites, an exceptionally high value compared to previous reports. Strong polymer colour changes were observed upon rebinding of certain anions, equivalent to those witnessed in solution, paving the way for application of such materials in anion sensing devices.

\section{Introduction}

Anions play an important role in many biological and chemical processes, thus neutral receptors able to selectively recognise and bind such species have attracted significant research interest in the past decades. ${ }^{1}$ The most common of them contain ureas, thioureas, pyrroles, amides, sulphonamides and squaramides as the binding groups, and the recognition mechanism is based on the formation of directional hydrogen bonds between the acidic $\mathrm{N}-\mathrm{H}$ functionality and the anionic guest in solution ${ }^{2}$ or supported on a polymer backbone. ${ }^{3}$ Furthermore, research efforts have focused on the development of such receptors with the added ability to signal the binding event by means of a change in colour or fluorescence upon complex formation. ${ }^{4}$ This is typically achieved by incorporation of "reporting" functional groups in close proximity to the binding centre.

Squaric acid, 3,4-dihydroxy-3-cyclobutene-1,2-dione, and its derivatives were first prepared by Cohen et al. ${ }^{5}$ and since then various synthetic protocols, ${ }^{6}$ their properties as anion or cation receptors ${ }^{7}$ and organo-catalysts ${ }^{8}$ have been studied extensively. More recently, squaramide based compounds have been used to transport anions across lipid bilayers and were shown to

School of Chemistry and Chemical Engineering, Queen's University Belfast, David Keir Building, Stranmillis Road, Belfast BT9 5AG, Northern Ireland, UK. E-mail: p.manesiotis@qub.ac.uk; Tel: +44 (0)28 9097 4515. Fax: +44 (0)28 90976524

$\dagger$ Electronic supplementary information (ESI) available: Analytical characterisation data, UV titration isotherms, Job plot, UV titration spectra and ${ }^{13}$ C CPMAS solid state NMR spectra. See DOI: 10.1039/c4tc01776e possess better anion-transport activities than analogous urea and thiourea based receptors. ${ }^{9}$ Despite the importance of squaramide receptors, to date there are very few reports of polymer bound squaramides. Rostami et al. ${ }^{10}$ employed a Lewis acid catalysed poly-condensation reaction to prepare fluorescent poly-squaramides, while Kasaplar et al. immobilised a chiral squaramide onto a polystyrene support using coppercatalysed azide-alkyne cycloaddition. ${ }^{11}$

Here, we report on the synthesis of a series of squaramide receptors with built-in polymerisable functionality that enables their incorporation as co-monomers in any chain-growth polymerisation reaction.

In contrast to previous reports, the polymerisable squaramides described in this article offer flexibility in terms of comonomer choice and composition of polymerisation feed, while they are compatible with the use of cross-linking monomers. The latter, enables their incorporation as anion or cation recognition elements or polymer supported organo-catalysts in e.g. gel-type resins and molecularly imprinted polymers (MIPs).

\section{Experimental section}

\section{General synthesis of squaramide monomers}

The protocol used for the synthesis of the polymerisable squaramides was adapted from a previously reported method. ${ }^{5}$ Symmetric squaramide 3 was prepared in $10 \mathrm{mmol}$ scale by reaction of 3,4-diethoxy-3-cyclobutene-1,2-dione 1 with 2.1 equivalents of 4 -vinylaniline in the presence of 0.2 equivalents of $\mathrm{Zn}(\mathrm{OTf})_{2}$ in ethanol, at room temperature. A light yellow 
precipitate was formed within the first 10 minutes however, the reaction was allowed to proceed for $24 \mathrm{~h}$. The precipitate was filtered, washed with ethanol and dried in vacuo. Nonsymmetric squaramides 4-8 were synthesised in two steps. Firstly, mono-substituted precursor 2 was prepared in $50 \mathrm{mmol}$ scale by reaction of 3,4-diethoxy-3-cyclobutene-1,2-dione 1 with an equimolar amount of 4-vinylaniline in the presence of 0.1 equivalents of $\mathrm{Zn}(\mathrm{OTf})_{2}$ in ethanol, at room temperature. The light yellow precipitate formed was filtered, washed with ethanol and dried in vacuo prior to further use. Then, $10 \mathrm{mmol}$ amounts of intermediate 2 were reacted with equimolar amounts of each corresponding aromatic or aliphatic amine to yield the final products. In this case, the reaction solvent was a mixture of toluene and DMF $9: 1$ and reactions were carried out under reflux.

This was necessitated by the fact that the reactivity of the used aromatic amines decreased with the presence of stronger EWGs. Thus, elevated temperatures and extended reaction times, up to $96 \mathrm{~h}$ for $\mathbf{6}$, were employed. On the contrary, aliphatic amines were significantly more reactive and all reactions were complete within $24 \mathrm{~h}$. In all cases, the final product was isolated by filtration of the precipitate

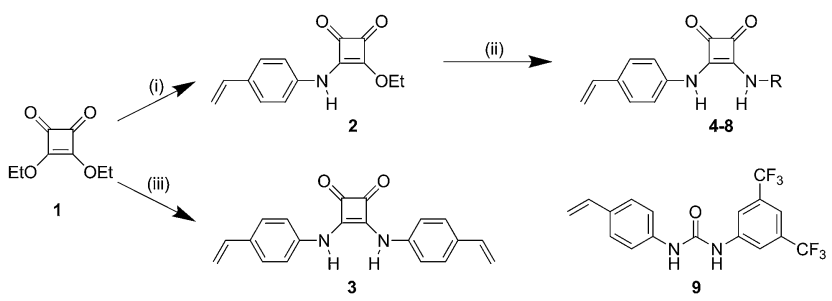

Fig. 1 Synthetic scheme for the preparation of polymerisable receptors 3-8 and structure of previously synthesised urea monomer 9 . (i) 1.1 eq. 4-vinylaniline, $20 \% \mathrm{~mol} \mathrm{Zn}(\mathrm{OTf})_{2}$, EtOH, r.t., 24 h; (ii) 1.1 eq. R$\mathrm{NH}_{2}, 20 \%$ mol Zn(OTf) 2 , toluene : DMF (9: 1), reflux, 24-96 h; (iii) 2.1 eq. 4-vinylaniline, $20 \% \mathrm{~mol} \mathrm{Zn}(\mathrm{OTf})_{2}$, EtOH, r.t., 24 h. 4: $\mathrm{R}=$ phenyl; 5 : $\mathrm{R}=3$-nitrophenyl; $6: \mathrm{R}=3$ 3-5is(trifluoromethyl)phenyl; 7: $\mathrm{R}=$ benzyl; 8: $\mathrm{R}=3,5$-bis(trifluoromethyl)benzyl. produced when the reaction was allowed to cool to room temperature, washed with hot toluene and dried in vacuo. Full analytical characterisation data for squaramides 3-8 can be found in the ESI section. $\dagger$

\section{Synthesis of imprinted polymers}

Tetrabutylammonium benzoate (TBAOBz) and monobasic tetrabutylammonium phosphate (TBAP) imprinted polymers were synthesised using a 1:1 ratio of functional monomer 3: template, plus 20 equivalents of the cross-linker ethyleneglycol dimethacrylate (EDMA). Thus, $0.048 \mathrm{~g}(0.13 \mathrm{mmol})$ of TBAOBz for P1, or $0.045 \mathrm{~g}(0.13 \mathrm{mmol})$ of TBAP for P2, and $0.042 \mathrm{~g}(0.13$ $\mathrm{mmol}$ ) of 3 were dissolved in $0.75 \mathrm{~mL}$ of DMSO followed by addition of $0.5 \mathrm{~mL}$ (2.65 mmol) of EDMA in a $10 \mathrm{~mL}$ screw-cap vial. For comparison purposes, a further set of polymers using previously reported urea monomer 9 were also synthesised in a similar fashion, albeit acetonitrile was used as the porogen. Thus, for P3, $0.048 \mathrm{~g}(0.13 \mathrm{mmol})$ of TBAOBz and $0.050 \mathrm{~g}(0.13$ $\mathrm{mmol}$ ) of 9 were dissolved in $0.75 \mathrm{~mL}$ of acetonitrile followed by addition of $0.5 \mathrm{~mL}(2.65 \mathrm{mmol})$ of EDMA. P4 was prepared identically to P3, with omission of the template. Lastly, P5, an imprinted polymer using monomer 6 (0.057 $\mathrm{g} ; 0.13 \mathrm{mmol})$ and TBAF (0.035 g; $0.13 \mathrm{mmol}$ ) as the template, EDMA (0.5 mL; 2.65 $\mathrm{mmol}$ ) in $0.75 \mathrm{~mL}$ DMSO was prepared in order to test the colour changing behaviour of the squaramide when polymerised. In all cases, pre-polymerisation solutions were degassed using $\mathrm{N}_{2}$ while cooling in an ice-bath for 5 minutes. Finally, $0.005 \mathrm{~g}$ of the free radical initiator 2,2'-azobis(2-methylpropionitrile) (AIBN) were added and the vials were sealed and placed in a water bath thermostated at $60{ }^{\circ} \mathrm{C}$ for 24 hours. Following polymerisation the resulting monoliths were smashed to coarse particles and washed with methanol using a Soxhlet apparatus and the polymers were manually ground and sieved collecting the $25-38 \mu \mathrm{m}$ fraction for further evaluation. P3, P4 and P5 were further analysed by solid state NMR in order to establish any influence of squaramide monomer 6 on polymer structure, compared to previously known monomer 9 (Fig. S6†). All acquired spectra as well as corresponding $T_{1}$ spin-
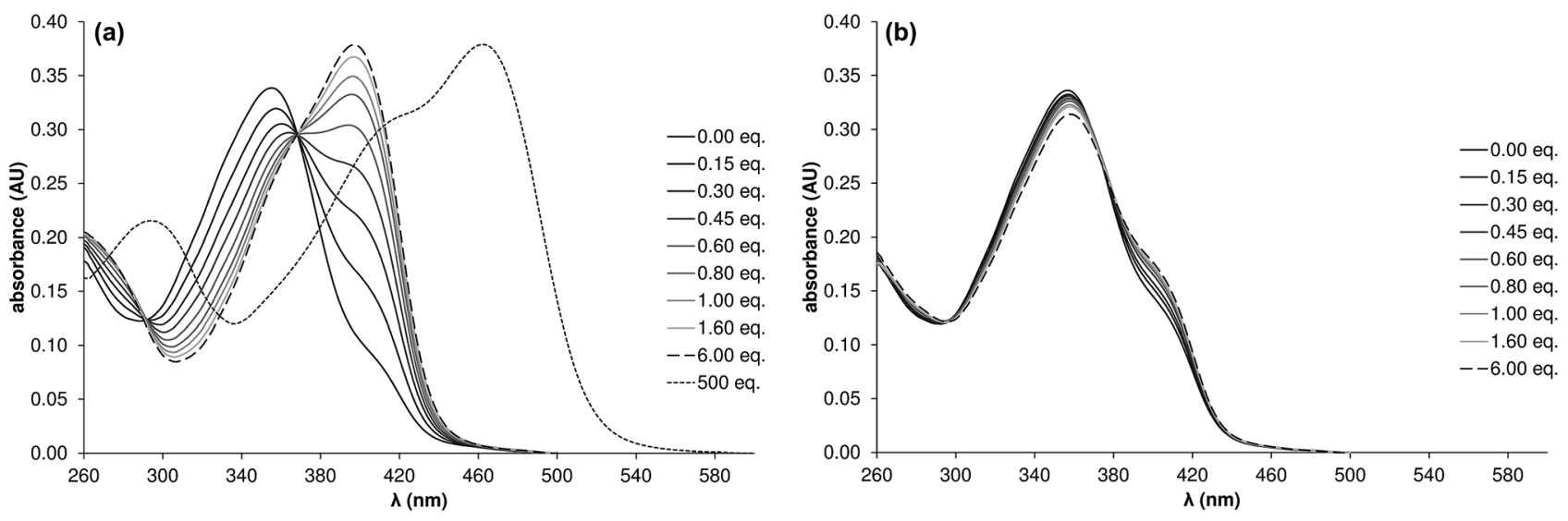

Fig. 2 UV-Vis spectra collected during the titration of receptor $6\left(1.0 \times 10^{-5} \mathrm{~mol} \mathrm{~L}^{-1}\right)$ with (a) TBAF and (b) TBAI, in DMSO. Corresponding spectra for titration of 6 with TBAOBz and TBAP available in ESI (Fig. S3†). 


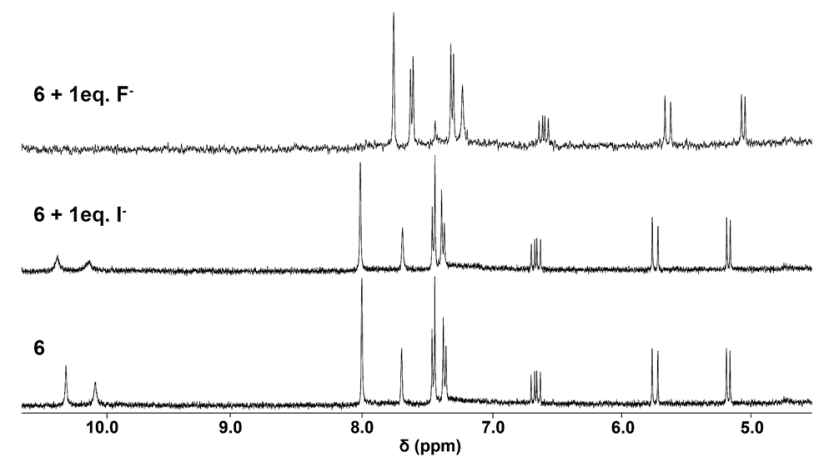

Fig. $3{ }^{1} \mathrm{H}-\mathrm{NMR}$ spectra $\left(400 \mathrm{MHz}, \mathrm{DMSO}-\mathrm{d}_{6}\right)$ of squaramide 6 (1.0 $\left.\mathrm{mmol} \mathrm{L}^{-1}\right)$ in the presence of equimolar amounts of TBAI or TBAF.

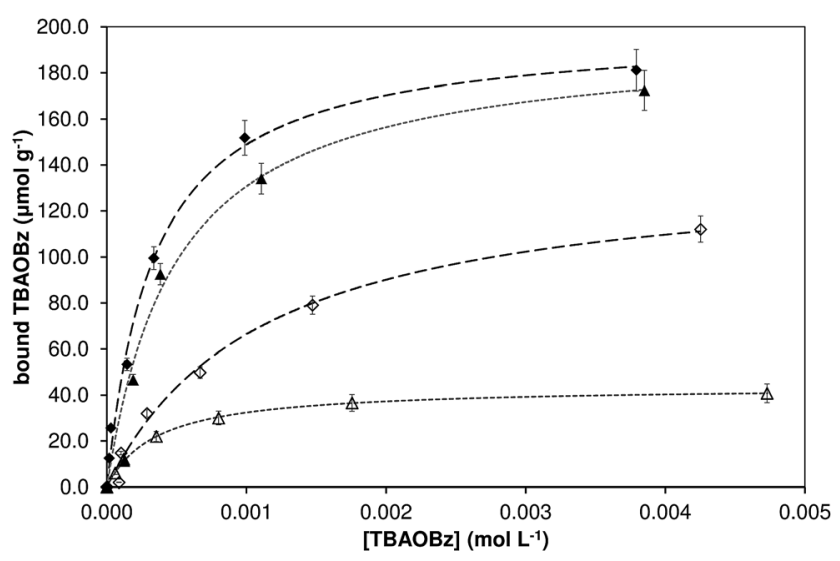

Fig. 4 Adsorption isotherms obtained by rebinding of TBAOBz on P1 (filled diamonds), P2 (open diamonds), P3 (filled triangles) and P4 (open triangles) in acetonitrile and corresponding Langmuir model fitted isotherms, calculated by non-linear regression.

lattice relaxation constants $(0.48-0.52$ seconds) were near identical, proving that the synthesised materials had very similar structural properties.

\section{Rebinding experiments}

$10 \mathrm{mg}$ of P1-P4 polymer particles were weighed each in a $2 \mathrm{~mL}$ glass vial and mixed with $1 \mathrm{~mL}$ of standard solutions of TBAOBz in concentrations of $0,0.1,0.2,0.5,1,2$ and $5 \mathrm{mmol} \mathrm{L}^{-1}$. After 24 hours, the concentration of unbound TBAOBz remaining in each supernatant solution was measured by UV spectroscopy, recording the absorbance at $260 \mathrm{~nm}$. All experiments were repeated in triplicate and the calculated averages and standard deviations were used to plot Fig. 4.

\section{Results and discussion}

The polymerisable receptors were synthesised via the high yielding method developed Rostami et al., ${ }^{6}$ using a Lewis acid, $\mathrm{Zn}(\mathrm{OTf})_{2}$, to catalyse the condensation of different substituted aromatic or aliphatic amines and 3,4-diethoxy-3-cyclobutene1,2-dione (1) (Fig. 1). Both symmetric and non-symmetric polymerisable squaramides were prepared, keeping 4- vinylaniline as the polymerisable component. Symmetric squaramide 3 was prepared in one step by reaction of $2.1 \mathrm{eq}$. of 4-vinylaniline with 1 eq. of 1 in ethanol, while non-symmetric receptors were prepared in two-steps. Initially, monosubstituted squaramide 2 was prepared and isolated, followed by further reaction with the corresponding substituted aniline or benzylamine to yield squaramide monomers 4-8. In all cases the product was isolated by precipitation from the corresponding reaction mixture in high purity and isolated yields of up to $85 \%$.

The binding strength of the polymerisable squaramides towards a series of common anions, in the form of tetrabutylammonium salts, was assessed by means of UV-Vis titrations in DMSO (Table 1). In agreement to previous reports, very high association constants were measured for all tested anions under polar competitive conditions, verifying our hypothesis that substitution of one "arm" of the receptor with a styryl group should not severely impact the overall performance of the receptors.

Among the aromatic substituted squaramides, the simplest, phenyl substituted receptor 4 showed the weakest association towards fluoride, phosphate and benzoate anions, followed by 3, the symmetric "cross-linker" squaramide. The effect of electron-withdrawing groups (EWG) attached to the aromatic substituents is demonstrated by the ten-fold increase in association constant $\left(K_{\mathrm{a}}\right)$ values of $\mathbf{5}$ and $\mathbf{6}$ against all tested anions, while de-protonation was observed when the latter were titrated against $>100$-fold excess of fluoride anions, whose strong basicity resulted in the abstraction of the most acidic squaramide NH proton.

As predicted, the association constants of the aliphatic substituted squaramides towards the tested anions were significantly weaker. Thus, the values obtained for receptors 7 and $\mathbf{8}$ were at least one order of magnitude lower than those of the corresponding aromatic receptors $\mathbf{4}$ and $\mathbf{6}$, clearly demonstrating the importance of conjugation in the binding performance. UV titration isotherms obtained during the study of the interaction of receptors 3-8 with fluoride and a Job plot verifying the $1: 1$ nature of the formed complexes are depicted in Fig. S1 and S2 respectively, in the ESI section, $\dagger$ together with

Table 1 Association constants $\left(K_{\mathrm{a}}, \mathrm{L} \mathrm{mol}{ }^{-1}\right)$ between the synthesised squaramide receptors and tetrabutylammonium salts of common anions, determined by UV titration experiments in DMSO. Host concentration kept constant at $1.0 \times 10^{-5} \mathrm{~mol} \mathrm{~L}^{-1}$

\begin{tabular}{lllll}
\hline Receptor & $\mathrm{C}_{6} \mathrm{H}_{5} \mathrm{COO}^{-}$ & $\mathrm{H}_{2} \mathrm{PO}_{4}{ }^{-}$ & $\mathrm{F}^{-}$ & $\mathrm{I}^{-}$ \\
\hline 3 & $1.0 \times 10^{5}$ & $3.6 \times 10^{5}$ & $6.7 \times 10^{5}$ & $<10^{3}$ \\
$\mathbf{4}$ & $5.1 \times 10^{4}$ & $6.5 \times 10^{4}$ & $4.1 \times 10^{5}$ & $<10^{3}$ \\
$\mathbf{5}$ & $7.8 \times 10^{5}$ & $4.0 \times 10^{5}$ & $>10^{6 a}$ & $9.8 \times 10^{4}$ \\
$\mathbf{6}$ & $9.1 \times 10^{5}$ & $5.1 \times 10^{5}$ & $>10^{6 a}$ & $3.8 \times 10^{5}$ \\
7 & $3.0 \times 10^{3}$ & $1.3 \times 10^{4}$ & $6.4 \times 10^{4}$ & $<10^{3}$ \\
$\mathbf{8}$ & $1.2 \times 10^{4}$ & $2.1 \times 10^{4}$ & $1.5 \times 10^{5}$ & $<10^{3}$ \\
$\mathbf{9}$ & $5.1 \times 10^{5}$ & $8.2 \times 10^{4}$ & $6.3 \times 10^{4}$ & $7.1 \times 10^{3}$
\end{tabular}

${ }^{a}$ Monomer de-protonation was observed upon addition of excess TBAF. 
characteristic UV titration spectra for all synthesised receptors (Fig. S3-S5†).

When compared with the previously reported 1-(3,5-bis(trifluoromethyl)phenyl)-3-(4-vinylphenyl)urea functional monomer (9) ${ }^{12}$ which has equivalent substitution to squaramide $\mathbf{6}$, the latter outperformed its counterpart by a factor of $\sim 100$ in the binding of fluoride, iodide and phosphate and was nearly twice as strong towards benzoate (Table 1). This could be explained by the proposed convergent orientation of the $\mathrm{NH}$ groups of the squaramide that favours interactions with localised negative charges, such as halide and phosphate anions, while the parallel orientation of the urea NHs favours interactions with delocalised anionic species such as carboxylates, with the formation of a pseudo 6-membered ring.

Furthermore, the stronger acidic nature of the squaramide $\mathrm{NH}$ protons compared to equivalent urea based receptors, results in their de-protonation at high concentrations of particularly basic anions, such as fluoride. This is verified by the appearance of their absorbance spectra (Fig. 2a) where upon addition of a large excess of TBAF (>100 eq.) to a solution of 6 , a strong colour change was observed associated with the appearance of new absorbance band at $470 \mathrm{~nm}$, indicative of charge transfer between the two species, and assigned to a deprotonation of the squaramide receptor $[\mathbf{6}-\mathbf{H}]^{-}$. Similar effects were not observed towards less basic anions such as iodide (Fig. 2b).

In order to further investigate whether de-protonation occurred at low fluoride concentrations, we prepared equimolar mixtures of 6 with fluoride and iodide and compared their ${ }^{1} \mathrm{H}-$ NMR spectra in DMSO- $\mathrm{d}_{6}$ with that of the free receptor. As seen in Fig. 3, the peaks assigned to the squaramide NHs disappear upon addition of 1 eq. fluoride, indicating strong hydrogen bonding, while a similar behaviour is not observed upon addition of iodide. Furthermore, the presence of fluoride has a significantly more pronounced effect on the other signals observed in the spectrum, corresponding to both aromatic as well as vinyl protons. This observation supports the hypothesis that receptor $\mathbf{6}$ was de-protonated only upon addition of great excess of fluoride anions and not at equimolar conditions. Hence, the spectral changes observed during the first part of the corresponding UV titration (Fig. 2a) can be attributed to hydrogen bonding, while abstraction of the most acidic $\mathrm{NH}$ proton, adjacent to the electron deficient aromatic ring, occurs when $>100$ eq. of fluoride are added. This observation is in agreement with previously reported studies by Amendola $e t$ al. as well as Kim et al. for EWG substituted (thio)urea and squaramide receptors. ${ }^{13}$

Having established that the newly synthesised squaramide monomers exhibit strong association towards a range of anionic species, it was decided to assess whether these can be utilised as polymer supported receptors. Thus, based on our previous expertise in the development of custom functional monomers for use in molecular imprinting, ${ }^{14}$ we opted to evaluate receptor 3 as a functional monomer in the imprinting of a model anion, tetrabutylammonium benzoate (TBAOBz). Molecularly Imprinted Polymers (MIPs) are highly cross-linked synthetic organic polymers that mimic the behaviour of natural receptors such as antibodies and enzymes, being able to recognise a targeted substance, the equivalent of an antigen or substrate..$^{15}$ Due to their highly cross-linked nature, MIPs are able to operate in purely organic as well as aqueous media, and have enhanced resistance to $\mathrm{pH}$, ionic strength, pressure and temperature, and an extended shelf-life compared to their natural counterparts.

MIPs are typically prepared by addition of a template in a pre-polymerisation mixture that contains a functional monomer, a porogenic solvent and a cross-linker, prior to initiation of the free-radical polymerisation reaction. This results in organisation of functionality and generation of size and shape complementary binding sites in the bulk of the resulting polymer network, such that these materials are subsequently able to selectively bind the templated compound in the presence of other competing analytes.

In this study, two novel squaramide based imprinted polymers were prepared and their binding performance was compared to polymers prepared using urea monomer 9. Thus, P1, an imprinted polymer prepared in the presence of TBAOBz, and P2, prepared in the presence of monobasic tetrabutylammonium phosphate (TBAP) were compared with a TBAOBz imprinted and control polymer, P3 and P4 respectively, prepared using urea monomer 9. It should be noted here that although MIP selectivity is typically assessed by comparison of an imprinted and a blank polymer, prepared in parallel but with omission of the template, as was the case with $\mathbf{P 3}$ and P4, P2 was itself an imprinted polymer, prepared however in the presence of a different template. The use of a compound that strongly associates with the functional monomer offered the added benefit of assisting dissolution of the polymerisable squaramide in the pre-polymerisation mixture, an effect which is often observed in MIP synthesis. Following grinding, sieving and washing of the resulting polymers to remove unreacted monomers and the corresponding templates, a rebinding experiment was setup in order to assess polymer selectivity and binding strength. Hence, a set amount (10 mg) of P1-P4 polymer particles was allowed to equilibrate with increasing concentrations of TBAOBz in acetonitrile. Binding isotherms correlating TBAOBz bound by the polymer $v s$. free TBAOBz were then plotted (Fig. 4). The experimental results were fitted to the Langmuir single binding site model and the calculated fitting parameters are presented in Table 2. Thus, P1 exhibited both the highest affinity and capacity towards its template, higher than that of P3, the urea based polymer. This observation vindicated the expectation that squaramides retain their stronger affinity for anionic species compared to equivalent ureas after polymerisation, despite the fact that P1 was synthesised using a moderately strong squaramide (3) as functional monomer and was prepared in DMSO, a polar solvent which is known to disrupt hydrogen bonding in the pre-polymerisation solution, instead of less polar acetonitrile used in the synthesis of P3. Control polymer P2, also exhibited strong binding for TBAOBz, albeit this was expectedly reduced, since this polymer contains cavities for TBAP, which has very different molecular shape and volume compared to TBAOBz. Lastly, P4, exhibited very low binding capacity for TBAOBz, attributed fully to non- 
Table 2 Affinity constants $\left(K_{\mathrm{a}}\right)$ and binding capacities $(q)$ of polymers $\mathrm{P} 1-\mathrm{P} 4$ for $\mathrm{TBAOBz}$ in acetonitrile, obtained by fitting the measured isotherms (Fig. 4) to the Langmuir adsorption model

\begin{tabular}{llll}
\hline Polymer & $K_{\mathrm{a}}\left(\mathrm{L} \mathrm{mol}^{-1}\right)$ & $q\left(\mu \mathrm{mol} \mathrm{g}^{-1}\right)$ & $R^{2}$ \\
\hline P1 & $3.0 \times 10^{3}$ & 198.9 & 0.9935 \\
P2 & $0.9 \times 10^{3}$ & 143.1 & 0.9895 \\
P3 & $2.0 \times 10^{3}$ & 194.8 & 0.9923 \\
P4 & $2.8 \times 10^{3}$ & 43.6 & 0.9991
\end{tabular}

specific interactions with the randomly distributed urea functional groups, which are still capable of hydrogen bonding to anionic species.

The high quality fits of the obtained isotherms to the Langmuir single binding site model, suggest that the synthesised imprinted materials exhibit a high degree homogeneity that can be attributed to the conversion of the strong $1: 1$ solution state complexes to embedded binding sites. A further important finding of this study however, becomes evident when the measured and maximum theoretical capacities of the synthesised polymers, in particular $\mathbf{P 1}$ and P3, are compared. Both polymers were prepared using the same amount of template in the pre-polymerisation solution, approximately $0.25 \mathrm{mmol}$ per gram of final material, thus their maximum theoretical capacity is approximately $250 \mu \mathrm{mol} \mathrm{g}{ }^{-1}$. As a result, the measured capacities of $\mathbf{P 1}\left(198.9 \mu \mathrm{mol} \mathrm{g}{ }^{-1}\right)$ and $\mathbf{P} 3\left(194.8 \mu \mathrm{mol} \mathrm{g} \mathrm{g}^{-1}\right)$ correspond to a yield of nearly $80 \%$ in accessible binding sites. This is an exceptionally high value, approaching quantitative conversion of monomer-template complexes to binding sites. It is noteworthy that commonly reported literature values correspond to an average $10-20 \%$ conversion of template to useable binding sites, while an excess of monomer typically used to promote complexation in the pre-polymerisation solution, often resulting in excessive non-specific binding by randomly distributed functionality. Apart from the weak interactions between commercially available monomers and targeted templates, such observations are commonly attributed to the mechanical stress imposed upon the polymers during grinding of polymer monoliths that impacts the integrity of the binding sites formed. However, the results presented here verify our position that custom functional monomer design, resulting in increased template binding strength while allowing the use of functional monomer(s) in stoichiometric quantities, is the key

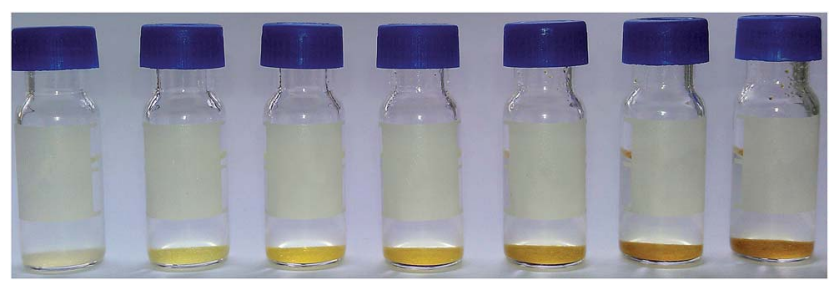

Fig. 5 Photograph of P5 polymer particles in the presence of increasing concentration of TBAF in acetonitrile. From left: $0,2,5,10$, $25,50,100 \mathrm{mmol} \mathrm{L}^{-1}$. parameter to ensure high affinity and high capacity of imprinted polymers, far exceeding the importance of polymerisation format or material mechanical treatment.

Further to the study of polymer capacity, a highly valuable property of the synthesised squaramide monomers was their ability to signal the presence of anionic species by colour change, an effect that was most pronounced in the case of fluoride binding by squaramide 6 . It was thus decided to investigate whether this behaviour was also maintained after polymerisation. To this end, $\mathbf{P 5}$, an imprinted polymer prepared using polymerisable squaramide 6 and TBAF as the template, was prepared similarly to the previously synthesised polymers. During preparation, it was observed that the pre-polymerisation solution obtained a dark orange colour, in agreement to previous observations made during UV-Vis titrations. This intense colour remained until the end of the reaction however, following a methanol wash the final material acquired a pale yellow hue, similar to that of the neat squaramide, indicating near complete removal of TBAF. More importantly, when P6 polymer particles were suspended in acetonitrile TBAF solutions of increasing concentration, a drastic colour change was immediately observed, proving that rapid and reversible association of TBAF took place, followed by de-protonation of the acidic squaramide $\mathrm{NH}$ groups resulting in darker orange appearance of the materials (Fig. 5). Interestingly, when TBAF was added to suspended particles of a similar polymer prepared in the presence of TBA iodide, the associated colour change was not as pronounced, proving that some binding selectivity was imparted to the polymers.

\section{Conclusions}

A series of novel polymerisable squaramides have been synthesised in moderate to high yields and their affinity towards common anions has been evaluated by means of UV-Vis titrations. Solution association constants ranged between $10^{3}$ and $>10^{6} \mathrm{~L} \mathrm{~mol}^{-1}$ and were influenced by substituent type and targeted anion. In the case of squaramide receptor 6 that contained the most acidic amide protons, evidence of deprotonation was supported by the appearance of a strong absorbance band at $470 \mathrm{~nm}$ when an excess of fluoride was added to the receptor. Imprinted polymers prepared using moderately strong squaramide receptor 3 were compared to equivalent materials prepared using a previously synthesised urea monomer. Both materials exhibited exceptionally high capacity for the model template TBA benzoate that corresponded to $80 \%$ conversion of monomer-template complexes to accessible binding sites. Lastly, the strong colour changes observed during the association of squaramide 6 with fluoride in solution, were also observed in the solid state upon addition of the anion to imprinted polymer particles.

The polymerisable squaramides presented here for the first time, pave the way for a plethora of polymer based anion binding and sensing applications, grace to their ability to copolymerise with vinyl monomers by robust, high yielding free radical polymerisation, as well as their ability to signal the presence of certain anions. Furthermore, the results obtained 
unambiguously prove that custom-made functional monomers, prepared from relatively inexpensive starting materials using simple synthetic steps, can result to exceptionally performing receptors when employed as functional monomers in molecular imprinting. These monomers are presently being investigated as receptors for selective binding and sensing of more complex substances of biological and environmental interest.

\section{Acknowledgements}

Financial support from Enterprise Ireland's Applied Research Enhancement Programme (Contract number: RE-2008-11) is gratefully acknowledged.

\section{Notes and references}

1 (a) P. A. Gale and T. Gunnlaugsson, Chem. Soc. Rev., 2010, 39, 3595; (b) R. Martínez-Máñez and F. Sancenón, Chem. Rev., 2003, 103, 4419; (c) J. L. Atwood, K. T. Holman and J. W. Steed, Chem. Commun., 1996, 1401.

2 (a) V. Amendola, L. Fabbrizzi, L. Mosca and F. P. Schmidtchen, Chem.-Eur. J., 2011, 17, 5972; (b) J. L. Sessler, S. Camiolo and P. A. Gale, Coord. Chem. Rev., 2003, 240, 17.

3 (a) A. Rostami and M. S. Taylor, Macromol. Rapid Commun., 2012, 33, 21; (b) S. Memon, E. Akceylan, B. Sap, M. Tabakci, D. M. Roundhill and M. Yilmaz, J. Polym. Environ., 2003, 11, 67; (c) R. Sakai, S. Okade, E. B. Barasa, R. Kakuchi, M. Ziabka, S. Umeda, K. Tsuda, T. Satoh and T. Kakuchi, Macromolecules, 2010, 43, 7406; (d) D. S. Jin, R. Ryong and S. J. Mu, Macromolecules, 1991, 24, 1727.

4 T. Gunnlaugsson, M. Glynn, G. M. Tocci, P. E. Kruger and F. M. Pfeffer, Coord. Chem. Rev., 2006, 250, 3094.

5 S. Cohen and S. G. Cohen, J. Am. Chem. Soc., 1966, 88, 1533. 6 (a) A. Rostami, A. Colin, X. Y. Li, M. G. Chudzinski, A. J. Lough and M. S. Taylor, J. Org. Chem., 2010, 75, 3983; (b) R. I. Storer, C. Aciro and L. H. Jones, Chem. Soc. Rev., 2011, 40, 2330.

7 (a) A. Frontera, J. Morey, A. Oliver, M. N. Pina, D. Quinonero, A. Costa, P. Ballester, P. M. Deya and E. V. Anslyn, J. Org.
Chem., 2006, 71, 7185; (b) D. Quinonero, A. Frontera, G. A. Suner, J. Morey, A. Costa, P. Ballester and P. M. Deya, Chem. Phys. Lett., 2000, 326, 247; (c) D. Quinonero, R. Prohens, C. Garau, A. Frontera, P. Ballester, A. Costa and P. M. Deya, Chem. Phys. Lett., 2002, 351, 115; (d) V. Ramalingam, M. E. Domaradzki, S. Jang and R. S. Muthyala, Org. Lett., 2008, 10, 3315.

8 (a) J. P. Malerich, K. Hagihara and V. H. Rawal, J. Am. Chem. Soc., 2008, 130, 14416; (b) J.-Y. Qian, C.-C. Wang, F. Sha and X.-Y. Wu, RSC Adv., 2012, 2, 6042; (c) W. Yang and D.-M. Du, Chem. Commun., 2011, 47, 12706; (d) W. Yang, J. Wang and D.-M. Du, Tetrahedron: Asymmetry, 2012, 23, 972.

9 N. Busschaert, I. L. Kirby, S. Young, S. J. Coles, P. N. Horton, M. E. Light and P. A. Gale, Angew. Chem., Int. Ed. Engl., 2012, $51,4426$.

10 A. Rostami, C. J. Wei, G. Guerin and M. S. Taylor, Angew. Chem., Int. Ed. Engl., 2011, 50, 2059.

11 (a) P. Kasaplar, P. Riente, C. Hartmann and M. A. Pericas, Adv. Synth. Catal., 2012, 354, 2905; (b) P. Kasaplar, C. Rodríguez-Escrich and M. A. Pericàs, Org. Lett., 2013, 15, 3498.

12 (a) A. Hall, P. Manesiotis, M. Emgenbroich, M. Quaglia, E. De Lorenzi and B. Sellergren, J. Org. Chem., 2005, 70, 1732; (b) P. Manesiotis, A. Hall, M. Emgenbroich, M. Quaglia, E. De Lorenzi and B. Sellergren, Chem. Commun., 2004, 2278.

13 (a) V. Amendola, G. Bergamaschi, M. Boiocchi, L. Fabbrizzi and M. Milani, Chem.-Eur. J., 2010, 16, 4368; (b) D. W. Kim, J. Kim, J. Hwang, J. K. Park and J. S. Kim, Bull. Korean Chem. Soc., 2012, 33, 1159.

14 (a) P. Manesiotis, Q. Osmani and P. McLoughlin, J. Mater. Chem., 2012, 22, 11201; (b) P. Manesiotis, A. Hall, J. Courtois, K. Irgum and B. Sellergren, Angew. Chem., Int. Ed. Engl., 2005, 44, 3902.

15 (a) Molecularly imprinted polymers. Man-made mimics of antibodies and their applications in analytical chemistry, ed. B. Sellergren, Elsevier Science B.V., Amsterdam, 2001, vol. 23; (b) G. Wulff, Angew. Chem., Int. Ed. Engl., 1995, 34, 1812; (c) G. Wulff, Chem. Rev., 2002, 102, 1; (d) J. L. Bowen, P. Manesiotis and C. J. Allender, Mol. Imprinting, 2013, 1, 35. 